\title{
N-Phthalyl-L-Tryptophan (RG108), like Clozapine (CLO), Induces Chromatin Remodeling in Brains of Prenatally Stressed Mice
}

\author{
Erbo Dong, Valentina Locci, Eleonora Gatta, Dennis R. Grayson, and Alessandro Guidotti \\ The Psychiatric Institute, Center for Alcohol Research in Epigenetics, Department of Psychiatry, College of Medicine, University \\ of Illinois at Chicago, Chicago, Illinois
}

Received June 26, 2018; accepted October 19, 2018

\section{ABSTRACT}

Schizophrenia (SZ), schizoaffective (SZA), and bipolar (BP) disorder are neurodevelopmental psychopathological conditions related, in part, to genetic load and, in part, to environmentally induced epigenetic dysregulation of chromatin structure and function in neocortical GABAergic, glutamatergic, and monoaminergic neurons. To test the above hypothesis, we targeted our scientific efforts on identifying whether the molecular epigenetic signature of postmortem brains of patients with SZ, SZA, and BP disorder are also present in the brains of adult mice born from dams prenatally restraint stressed (PRS) during gestation. The brains of PRS mice, which are similar to the brains of patients with SZ and BP disorder, show an $\sim 2$-fold increased binding of DNMT1 to psychiatric candidate promoters (glutamic acid decarboxylase 67, Reelin, and brain-derived neurotrophic factor), leading to their hypermethylation, reduced expression, as well as the behavioral endophenotypes reminiscent of those observed in the above psychiatric disorders. To establish whether clozapine (CLO) produces its behavioral and molecular action through a causal involvement of DNA methylation/demethylation processes, we compared the epigenetic action of CLO with that of the DNMT1 competitive inhibitor N-phthalyl-Ltryptophan (RG108). The intracerebroventricular injection of RG108 (20 nmol/day per 5 days), similar to the systemic administration of CLO, corrects the altered behavioral and molecular endophenotypes that are typical of PRS mice. These results are consistent with an epigenetic etiology underlying the behavioral endophenotypic profile in PRS mice. Further, it suggests that PRS mice may be useful in the preclinical screening of antipsychotic drugs acting to correct altered epigenetic mechanisms.

\section{Introduction}

The development of new and effective drugs that also show reduced toxicity to treat the complex symptomatology of schizophrenia (SZ), schizoaffective (SZA), and bipolar (BP) disorder is impeded by the lack of pertinent diagnostic tools to assess disease prodromes, progression severity, and beneficial drug responses. Additional fundamental hurdles to the identification of new drugs to effectively treat SZ, SZA, and BP disorder include an insufficient appreciation of the causal and developmental mechanisms underlying the symptoms characteristic of these diseases. Moreover, the inability to reproduce the complex nature of these disorders in laboratory animals is an additional barrier to drug discovery.

SZ and related psychiatric disorders are neurodevelopmental disorders with a significant epigenetic pathophysiology brought about by exposure to prenatal or perinatal

This work was funded by grants provided by the National Institute on Alcohol Abuse and Alcoholism [P50AA022538], National Institute of Mental Health [R01 MH093348], [R01 MH101043], and the Center for Clinical and Translational Science Pilot Translational and Clinical Studies Program [CCTS 2017-04].

https://doi.org/10.1124/mol.118.113415. environmental insults, including stress, drugs, maternal infection, and trauma (Bock et al., 2015; Khashan et al., 2008; van Os et al., 2010; Markham and Koenig, 2011; McGowan et al., 2011; Zhang et al., 2013; Cao-Lei et al., 2015, 2016; Labouesse et al., 2015) and a variable genetic load (Weinberger 1987; Fatemi and Folsom, 2009; Schizophenia Working Group of the Psychiatric Genomics Consortium, 2014; Sekar et al., 2016). Epigenetic studies indicate that aberrant DNA methylation profiles likely play an important neurodevelopmental role in psychiatric disease pathogenesis and as a target for drug discovery (Mill et al., 2008; Ptak and Petronis, 2010; Grayson and Guidotti, 2013). We have previously established that prenatally restraint-stressed (PRS) mice exhibit SZ-like endophenotypic characteristics that can be used to identify robust and efficacious antipsychotic drugs that normalize epigenetic mechanisms (Dong et al., 2016).

The frontal cortices of PRS mice, similar to those of SZ, SZA, and BP postmortem brains, exhibit an epigenetic profile that includes an increase in Dnmt1 binding to the glutamic acid decarboxylase 67 (Gad1), Reelin (Reln), and brain-derived neurotrophic factor (Bdnf) promoters (Matrisciano et al., 2013; Dong et al., 2015b, 2018) and an enrichment of 5-methylcytosine $(5 \mathrm{mC})$ and 5-hydroxymethylcytosine at

ABBREVIATIONS: ADORA1, adenosine receptor A1; BDNF, brain-derived neurotrophic factor; BP, bipolar; CLO, clozapine; DMSO, dimethylsulfoxide; DRD2, dopamine receptor D2; Gad1, glutamic acid decarboxylase 67; H3, histone3; HAL, haloperidol; H3Kac, lysine acetylated histone 3; H3K4me , H3 trimethyl lysine-4; H3K27me3, trimethylated lysine 27 histone 3; HTR, 5-hydroxytryptamine receptor; 5mC, 5methylcytosine; NS, nonstressed; PCR, polymerase chain reaction; PFC, prefrontal cortex; PND, postnatal day; PRS, prenatal restraint-stressed; Reln, Reelin; RG108, N-phthalyl-L-tryptophan; RIS, risperidone; SZ, schizophrenia; SZA, schizoaffective; VEH, vehicle; VPA, valproic acid. 
these same promoter regions (Dong et al., 2015a, 2016). These increases in promoter methylation are associated with reduced levels of synapse-associated proteins, such as Bdnf and Reln (Dong et al., 2015a, 2016) and behavioral deficits that resemble aspects of the positive and negative symptoms (Jones et a., 2011), and the cognitive impairment, detected in patients with SZ, SZA and BP disorder (Murmu et al., 2006; Lisman et al., 2008; Fine et al., 2014; Ruzicka et al., 2015). Interestingly, the enrichment of $5 \mathrm{mC}$ at the $B d n f, G a d 1$, and Reln promoters in the medial prefrontal cortex (PFC) and hippocampus of PRS mice correlates with the behavioral endophenotypes (Dong et al., 2015a, 2016). Although there is general agreement that altered epigenetic mechanisms play an important role in the etiopathogenesis of SZ and BP disorder (Guidotti et al., 2011; Grayson and Guidotti, 2013), little is known regarding the effects of drugs, including typical and atypical antipsychotic drugs, on the epigenetic modifications of GABAergic, glutamatergic, or monoaminergic neurons in the neocortices of patients with $\mathrm{SZ}$ and $\mathrm{BP}$ disorder.

In the current study, we first replicated previous results on the hypermethylation of Bdnf, Reln1, and Gad1 promoters in PRS mice. To validate the PRS mouse as a reasonable epigenetic animal model for studying the actions of specific groups of neuroleptic drugs acting on altered DNA methylation, we compared the effects of a typical antipsychotic drug, haloperidol (HAL) [acting at dopamine receptor D2 (DRD2)], and two atypical antipsychotics: clozapine (CLO) [active at various receptors including 5-hydroxytrptamine receptor (HTR) 1A, HTR2A, HTR2C, and adenosine receptor A1 (ADORA1)] and risperidone (RIS) (active at DRD2, HTR2A, HTR2C, and ADORA1 receptors) (Table 1) (Jarskog et al., 2007; Meltzer, 2012) with the effects of $N$-phthalyl-L-tryptophan (RG108), a recently identified compound that selectively targets the catalytic domain of DNMT1 (for structure, see the study by Asgatay et al., 2014).

\section{Materials and Methods}

Animals and PRS Procedure. All procedures were performed according to guidelines of the National Institutes of Health for animal research and were approved by the Institutional Animal Care Committee of the University of Illinois at Chicago. Timed pregnant mice (Swiss albino ND4) were obtained from Envigo (Indianapolis, IN). Pregnant dams were individually housed with a 12-hour light/dark cycle and food and water available ad libitum. The pregnant mice were left to habituate to the new housing conditions for 1 to 2 days and were then randomly assigned to nonstressed (NS) or stressed groups. NS dams were left undisturbed throughout gestation, whereas stressed dams were subjected to repeated episodes of restraint stress, as described previously (Dong et al., 2016). Experiments were performed on mice between PNDs 60 and 85. Male mice were chosen for the experiments because they exhibit more robust and consistent stereotyped behavior, reduced social interactions, prepulse inhibition, and fear-conditioning deficits than female mice. However, in a recent study (Dong et al., 2018) we also established that female PRS mice show more anxiety than male mice.

Drug Treatment. CLO (Sandoz Pharmaceuticals, Princeton, NJ), HAL (Millipore-Sigma, St. Louis, MO), and RIS (Tocris Bioscience, Minneapolis, MN) were dissolved as previously described (Dong et al., 2008). RG108 was dissolved in 10\% dimethylsulfoxide (DMSO).

Drugs (CLO $5 \mathrm{mg} / \mathrm{kg}$, HAL $1 \mathrm{mg} / \mathrm{kg}$, and RIS 0.5 and $5 \mathrm{mg} / \mathrm{kg}$ ) were administered to PRS and NS mice at PND 60-75 subcutaneously twice daily for 5 consecutive days. It is noteworthy that drugs used in this study elicited catalepsy (HAL), and sedation (CLO, RIS) which are maximal after approximately 1 hour but disappear after 18 hours of washout when the animals were resubmitted to the behavioral tests.

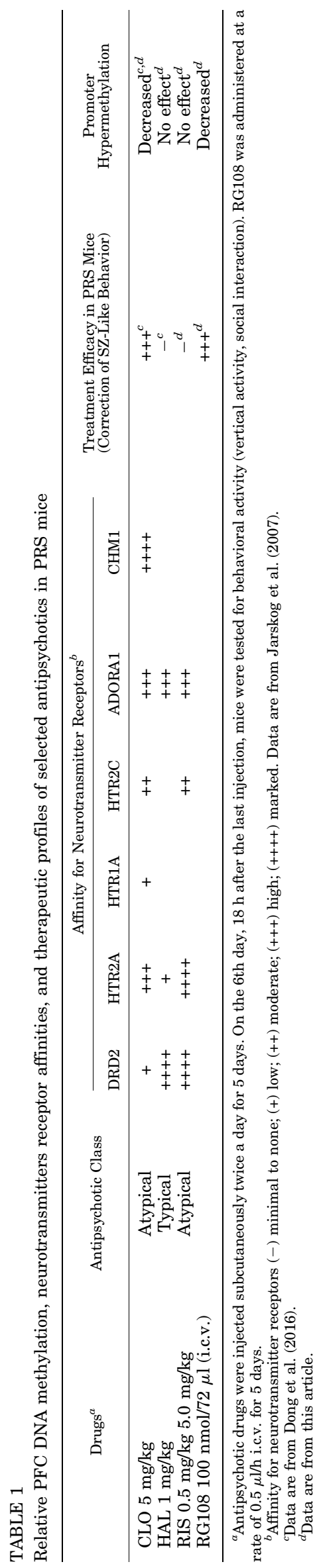


It should be noted that drug doses and duration of treatment were chosen on the basis of preliminary studies showing that CLO $(5 \mathrm{mg} / \mathrm{kg})$ requires multiple days of treatment to improve behavioral deficits and elicits demethylation of hypermethylated Reln promoters in mice treated protractedly with large doses of methionine (Dong et al., 2008; Guidotti et al., 2009, 2011; Alachkar et al., 2018).

RG108 is a noncompetitive antagonist of DNMT1 activity (Asgatay et al., 2014). Because RG108 does not enter the brain at sufficient concentrations due to the blood-brain barrier, it was administered by intracerebroventricular infusion using an osmotic mini pump connected with a brain infusion kit (Alzet; Durect Corporation, Cupertino, CA). The pump delivered a $0.5 \mu \mathrm{l} / \mathrm{h}$ solution containing $100 \mathrm{nmols}$ of RG108 in 10\% DMSO in $72 \mu \mathrm{l}$ for 5 days. Vehicle (VEH; 10\% DMSO alone) was administered to corresponding control groups using a similar pump delivery regimen. Behavioral tests were conducted 18 hours after the last administration of RG108, antipsychotic drug, or VEH.

Behavioral Tests. Stereotyped rearing activity and social interactions were examined in succession on the same day between 10:00 AM and 3:00 PM. We selected PNDs 60-75 for behavioral testing because at this postnatal time, the performance of the offspring was more reproducible and stable than the performance measured at earlier developmental time points (Matrisciano et al., 2013).

Stereotype Rearing Activity. A computerized Animal Activity Monitoring System with VersaMax software (AccuScan Instruments, Columbus, $\mathrm{OH}$ ) was used for the quantification and tracking of vertical activity in mice, as described previously (Matrisciano et al., 2013). The total number of interruptions of the vertical sensors was taken as a measure of stereotyped behavior. Activities were recorded for 15 minutes.

Social Interaction. Social interaction was measured using the "Thee-Chambers Apparatus" described in detail previously (Dong et al., 2016).

Social approaches were defined as the ratio of the sniffing time for the wire cup enclosing the stranger mouse versus that for the empty cup. The reliability of the measurements was assessed by correlating the scores of different raters.

Experimental Design. For these experiments, we used three independent cohorts of mice. In the first cohort, we compared CLO to HAL in six groups of mice: Group 1, NS mice receiving VEH; Group 2, NS mice receiving CLO; Group 3, NS mice receiving HAL; Group 4, PRS mice receiving VEH; Group 5, PRS mice receiving CLO; and Group 6, PRS receiving HAL. In the second cohort, we have: Group 1, NS mice receiving VEH; Group 2, NS mice receiving RIS $(0.5 \mathrm{mg} / \mathrm{kg})$; Group 3, NS mice receiving RIS ( $5 \mathrm{mg} / \mathrm{kg}$ ); Group 4, PRS mice receiving VEH; Group 5, PRS mice receiving RIS ( $0.5 \mathrm{mg} / \mathrm{kg})$; and Group 6, PRS receiving RIS $(5 \mathrm{mg} / \mathrm{kg})$. In the third cohort we have: Group 1, NS mice receiving VEH; Group 2, NS mice receiving RG108; Group 3, PRS mice receiving VEH; and Group 4, PRS mice receiving RG108 $(100 \mathrm{nmol} /$ day per 5 days i.c.v.). Because these experiments are labor intensive and time consuming, we used a different cohort for each drug tested.

For the statistical analyses, to reduce the effect of variability among cohorts, results of CLO, HAL, RIS, and RG108 treatments were compared with the values of the respective controls and were expressed as the percentage of VEH-treated control mice.

PFC Dissection. A Jacobowitz mouse brain slicer matrix was used for the dissection of the cortex. Starting from the frontal lobe (anteroposterior, $4.28 \mathrm{~mm}$ ), thee slices of $1 \mathrm{~mm}$ were mounted on a cold coverslip and disks of $1.5 \mathrm{~mm}$ in diameter were punched from the frontoparietal motor and sensory cortices. Using this procedure, we obtained $\sim 20 \mathrm{mg}$ of tissue for DNA/RNA studies (Impagnatiello et al., 1996).

Real-Time Polymerase Chain Reaction. The polymerase chain reaction (PCR) was carried out using an Applied Biosystems RealTime PCR System (Thermo Fisher Scientific, Foster City, CA) using SYBR Green Master Mix (Fermentas, Glen Burnie, MD). For additional details, see the study by Dong et al. (2016).
Methylated DNA Immunoprecipitation. Methylated DNA at the Gad1, Reln, and Bdnf IX promoters were measured using immunoprecipitation (MagMeDIP), as described by the manufacturer (Diagenode, Denville, NJ). We used immunoprecipitation with specific antibodies to distinguish $5 \mathrm{mC}$ from the approximately $20 \%$ of methylcytosines in the brain that are 5-hydroxymethylcytosine (Wen et al., 2014). The percentage of methylated versus unmethylated promoter was calculated using the following equation: \% [(meDNA -

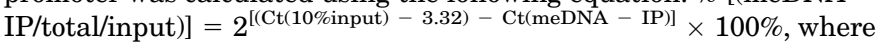
IP is immunoprecipitation and meDNA is methylated DNA. The efficiency of methyl DNA immunoprecipitation was determined by quantitative reverse-transcription polymerase chain reaction using internal positive and negative DNA controls (methylated/hydroxymethylated and unmethylated DNA), as well as control primers for the testis-specific H2B histone gene (TSH2B; which is methylated in all somatic cells but not in testis), and glyceraldehyde-3-phosphate dehydrogenase promoter, which is poorly methylated. Data obtained with internal positive and negative DNA controls indicate a specificity of $\sim 96 \%$ [Specificity $\%=1-$ (enrichment unmethylated/enrichment methylated) $\times 100$ ]. No PCR signal was obtained when using an IgG control (Gatta et al., 2017).

Chromatin Immunoprecipitation Assays. We performed chromatin immunoprecipitation assays for DNMT1 binding based on protocols previously described (Dong et al., 2016). The percentages of immunoprecipitated DNA were calculated as described for methyl DNA immunoprecipitation assay. Chromatin immunoprecipitation grade DNMT1-specific antibodies (Imgenex, San Diego, CA) were used to coprecipitate cross-linked chromatin as previously described (Dong et al., 2016). No PCR signal was detected using an IgG control sample.

Western Blotting. All extraction procedures were carried out with an EpiQuik Total Histone Extraction Kit at $4^{\circ} \mathrm{C}$, according to manufacturer (Epigentek, Farmingdale, NY) instructions. To ensure similar protein loading, the total protein concentration of these extracts was determined by using the bicinchoninic acid assay method (Pierce BCA Protein Assay Kit; Thermo Fisher Scientific, Waltham, MA) using bovine serum albumin as a standard.

Samples were diluted in Laemmli buffer, denatured at $75^{\circ} \mathrm{C}$ for 10 minutes, run on 4\%-12\% Bis-Tris gel (Thermo Fisher Scientific), and blotted onto polyvinylidene difluoride membranes (MilliporeSigma). Membrane preparations were incubated overnight at $4^{\circ} \mathrm{C}$ with anti-histone antibodies diluted in 5\% dry milk, $0.1 \%$ Tween 20 in Tris-buffered saline, washed, and further processed with horseradish peroxidase-conjugated secondary antibodies (GE Healthcare, Chicago, IL). Immunocomplexes were revealed by enhanced chemiluminescence with an Immobilon Western Chemiluminescent HP Substrate Kit (Merck, Burlington, MA) by using the LI-COR (Lincoln, NE) ODYSSEY System and analyzed with Image Studio 5.2 (iS5.2) software (LI-COR).

To profile the pattern of histone tail modifications, we used the following antibodies: polyclonal anti-histone3 (H3) (1:100; MilliporeSigma); polyclonal anti-H3Kacetylated (H3Kac) (1:1000; MilliporeSigma); monoclonal anti-H3 dimethyl K9 (1:1000; Abcam, Cambridge, MA); monoclonal anti-H3 trimethyl lysine-4 (H3K4me ${ }^{3}$ ) (1:1000; Cell Signaling Technology, Danvers, MA), and monoclonal anti-histone H3 trimethyl lysine-27 (H3K27me ${ }^{3}$ ) (1:500; Cell Signaling Technology). The antibodies were highly specific. After immunoblotting, only a single reactive band was detected, and the corresponding molecular weight was that of expected modified histone. In addition, preadsorbing antibody with the corresponding synthetic peptides resulted in a complete loss of signal. In all blots, the optical density of the H3Kac, $\mathrm{H} 3 \mathrm{~K} 4 \mathrm{me}^{3}$, and $\mathrm{H} 3 \mathrm{~K} 27 \mathrm{me}^{3}$ bands were quantified and normalized relative to the optical density of the correspondent H3 band. The antiH3 antibody detects both unmodified and modified H3 variants (total H3). Samples from VEH- and drug-treated animals were analyzed in the same immunoblot, and values were calculated relative to VEHtreated mice.

Statistical Analysis. Results are expressed as the mean \pm S.D. and are presented as a percentage of respective NS mice receiving 
VEH. Experimental differences were assessed by two-way analysis of variance (ANOVA) followed by Tukey's post hoc comparisons, using Predictive Analytics SoftWare version 18 (SPSS, Chicago, IL). The criterion for significance was $P<0.05$, two tailed. Correlation analyses were performed using Pearson's correlation test. Western blotting data were analyzed using Student's $t$ test.

\section{Results}

CLO, but neither HAL nor RIS, Corrects the Behavioral Endophenotype in PRS Mice. Table 1 reports the high-affinity neurotransmitter receptor profile of three antipsychotic drugs, CLO, HAL, and RIS, each of which was tested for the ability to correct the behavioral endophenotypes and the molecular abnormalities in PRS mice. We previously established that PRS mice exhibit several behavioral alterations, including increased stereotypy, deficits in social interaction time, hyperlocomotor activity in an open field, and deficits in prepulse inhibition of acoustic startle (Matrisciano et al., 2013). The behavioral studies with PRS mice include increased vertical activity (stereotyped behavior) and reduced social interaction. These tests were selected because they produce limited distress for the animals that are then used for subsequent biochemical studies (Dong et al., 2016). For the behavioral experiments, young adult (PND 60-75), NS, and PRS male mice were treated for 5 days with either VEH or the drug under study and then withdrawn for 18 hours to eliminate any acute effects of antipsychotic drugs on behavior. As shown in Fig. 1, we replicated previous reports (Dong et al., 2016) that CLO corrects the behavioral endophenotypes associated with PRS mice, whereas HAL fails to do so. In addition, we observed that RIS $(0.5$ or $5 \mathrm{mg} / \mathrm{kg}$ ) was ineffective at reducing the increased stereotyped behavior (Fig. 1A) or relieving the social interaction deficit of PRS mice (Fig. 1B). To establish whether CLO produces its behavioral effects via a causal control of DNMT-mediated promoter methylation, we compared the epigenetic action of CLO with that of the DNMT1 competitive inhibitor RG108 in PRS mice. The intracerebroventricular infusion of RG108 (100 nmol/day for 5 days), similar to CLO ( $5 \mathrm{mg} / \mathrm{kg}$ twice a day for 5 days), corrects the behavioral endophenotypes (Fig. 1).

CLO, but neither HAL nor RIS, Corrects the Increased DNMT1 Binding and 5mC Promoter Hypermethylation of SZ-Associated Genes in PRS Mice. To establish the concept that Bdnf promoter methylation modulates the expression of its mRNA, we tested whether HAL or RIS could correct the local DNA methylation enrichment at cytosines proximal to the transcriptional start site of the Bdnf-ix promoter in the dorsal hippocampus of PRS mice. We observed that the enrichment of cytosine methylation at the Bdnf-ix promoter (Fig. 2) was not reduced in either PRS or NS mice treated with HAL or RIS. In contrast, in a replication of our previous study (Dong et al., 2016) with new cohorts of PRS and NS mice, we showed that CLO corrects both the increased Dnmt1 binding (Fig. 3A) and $5 \mathrm{mC}$ enrichment (Fig. 3B) to selected target promoters (Bdnf-ix, Gad1, and Reln). Collectively, these data suggest that CLO, unlike HAL or RIS, may limit DNA methylation, either by reducing the expression of Dnmts, or more likely by interfering with Dnmt1-DNA binding capability (Grayson and Guidotti, 2013, 2016) or Dnmt1 enzymatic activity.
A

Stereotype behavior

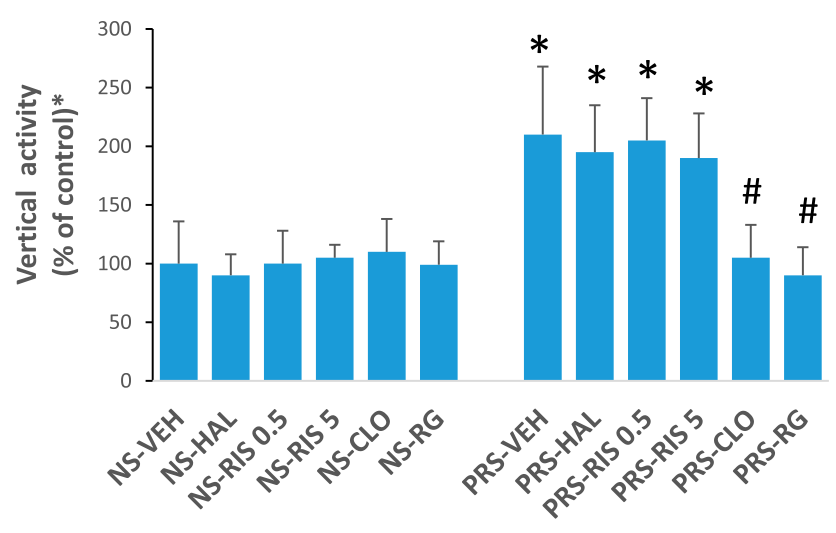

B

Social interaction

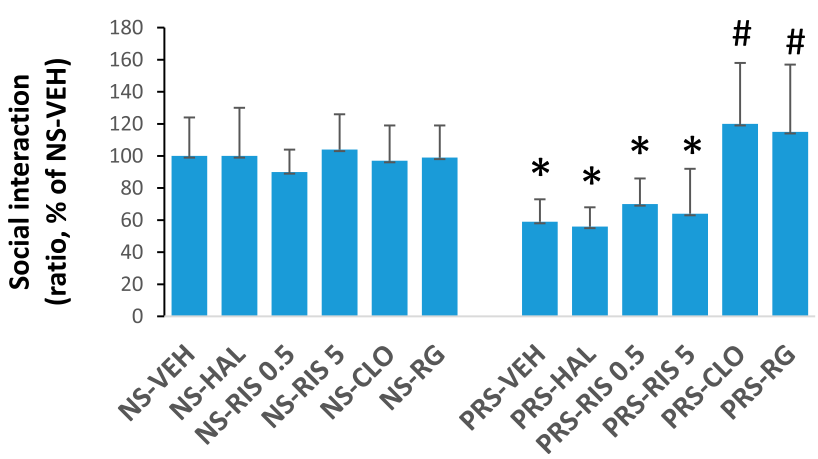

Fig. 1. Increased stereotype behavior (A) and social interaction deficits (B) in PRS mice is reversed by treatment with CLO but not HAL or RIS. Sixty-day-old PRS or NS male mice were treated subcutaneously twice daily, for 5 days with VEH, $5 \mathrm{mg} / \mathrm{kg}$ CLO, $1 \mathrm{mg} / \mathrm{kg} \mathrm{HAL}$, and 0.5 or $5 \mathrm{mg} / \mathrm{kg}$ RIS. Stereotypic behavior and social interaction were measured 18 hours after the last antipsychotic drug treatment. The data are expressed as the mean \pm S.D. of five to six mice per group. Because we used different VEH groups for each drug used, to compare the effect of the drugs, the values are expressed as the percentage of the corresponding NS groups receiving VEH. (A) Two-way ANOVA, group $\times$ treatment effect $F_{5,76}=4.23, P=0.002$ followed by Tukey's post hoc analysis. ${ }^{*} P<0.05$ when PRS mice are compared with the respective NS VEH-treated mice. $\# P<0.05$ when PRS mice treated with CLO and RG108 are compared with PRS mice receiving $\mathrm{VEH}$. Average values of vertical activity in NS mice were $280 \pm 60$. (B) The social interaction activity (ratio of sniffing time to the wire cup with a stranger mouse vs. the wire cup without the stranger mouse) was measured in the same mice used in (A) about 18 hours after the last antipsychotic drug treatment. The data are expressed as the mean \pm S.D. of five to six mice per group. Two-way ANOVA, group $\times$ treatment effect $\left(F_{5,88}=3.45, P=0.007\right)$ followed by Tukey's post hoc analysis. $* P<0.05$ when PRS mice are compared with the respective NS VEH-treated mice. $\# P<0.05$ when PRS mice treated with CLO and RG108 are compared with PRS mice receiving VEH. The average values of social interaction ratio in five groups of NS mice are $1.57 \pm 0.43$.

RG108, Like CLO, Corrects the Behavioral Phenotype and Alterations in Target Promoters in the PFC of PRS Mice. We also established that RG108, administered intracerebroventricularly, corrects the methylation enrichment as well as the increased binding of DNMT1 to the Gad1, Reln, and Bdnf-ix promoters in the cortex of PRS mice (compare Figs. 3 and 4). An important observation of this study is that CLO, like valproic acid (VPA) (Dong et al., 2016), has no effect on either the behavior or promoter methylation or the expression of mRNA and protein that we studied when administered to control mice or when injected only one time, 18 hours before testing. A possible explanation for the failure 


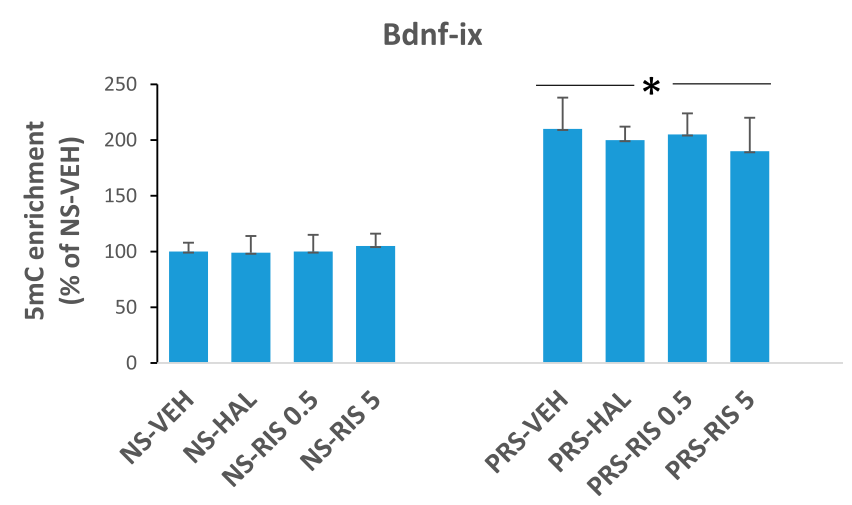

Fig. 2. The increased levels of $5 \mathrm{mC}$ at Bdnf-IX promoter region in the frontal cortex of PRS mice are not corrected by HAL or RIS. The enrichment of $5 \mathrm{mC}$ was measured on the 6th day, $\sim 18$ hours after the last day treatment. The data are expressed as the mean \pm S.D. of five to six mice per group. Two-way ANOVA, group effect for $5 \mathrm{mC}$ : Bdnf-IX $\left(F_{1,72}=318,{ }^{*} P<0.001\right)$. Average value of $5 \mathrm{mC}$ levels in three groups of NS mice is about $1 \%$ of the input.

of CLO to demethylate target promoters in NS mice is that the dynamic state of DNA methylation may be accelerated in PRS mice when compared with the NS mice. Hence, the steadystate methylation process may be very sensitive to short-term inhibition in PRS mice.

The Restorative Action of CLO on Altered Epigenetic Mechanisms Is not Mediated by Histone-PostTranslational Covalent Modifications. The restorative effects of CLO on the molecular and behavioral endophenotypes of PRS mice can be duplicated by the histone deacetylase inhibitor VPA (Tremolizzo et al., 2005; Simonini et al., 2006) directly acting on histone acetylation levels. Of note, VPA increases histone acetylation levels in both NS and PRS mice (Tremolizzo et al., 2005). In addition, there is one report (Huang et al., 2007) that demonstrates an increase in the levels of $\mathrm{H} 3 \mathrm{~K} 27 \mathrm{me}^{3}$ in the cortex of mice treated chronically with (CLO $5 \mathrm{mg} / \mathrm{kg}$, daily for 21 days). Therefore, we tested whether single (1 hour before) or repeated injections of CLO ( $5 \mathrm{mg} / \mathrm{kg}$ twice a day for 5 days and followed by an 18-hour withdrawal period) alter the levels of H3Kac, H3K4me ${ }^{3}$, or H3K27 $\mathrm{me}^{3}$ in NS mice. In Fig. 5, we show representative images of the Western blot for H3K27me $\mathrm{m}^{3}$. The values were normalized to the levels of nonmethylated or nonacetylated H3. No significant differences in the levels of these histone variants were detected in the medial $\mathrm{PFC}$ of mice receiving CLO. For example, the levels of H3Kac in the PFC are $110 \% \pm$ $10 \%$ and $101 \% \pm 16 \%$ of control $(n=5)$ in mice that underwent single treatment and repeated treatment with CLO, respectively. The levels of $\mathrm{H} 3 \mathrm{~K} 27 \mathrm{me}^{3}$ were $127 \% \pm 7.6 \%$ of controls, and those of $\mathrm{H} 3 \mathrm{~K} 4 \mathrm{me}^{3}$ were $80 \% \pm 18 \%$ of controls $(n=5$, nonsignificant) in PFCs of mice treated for 5 days with CLO.

\section{Discussion}

In this study, we describe a neurodevelopmental, epigenetic mouse model for psychiatric disease (the in utero stressed mouse or PRS mouse) that in the cerebral cortex expresses an increased DNMT1 binding and 5-cytosine hypermethylation of promoters (Reln, Gad1, and Bdnf) that represent the core epigenetic pathophysiological signature underlying SZ, SZA, and $\mathrm{BP}$ disorders. This model responds to the administration
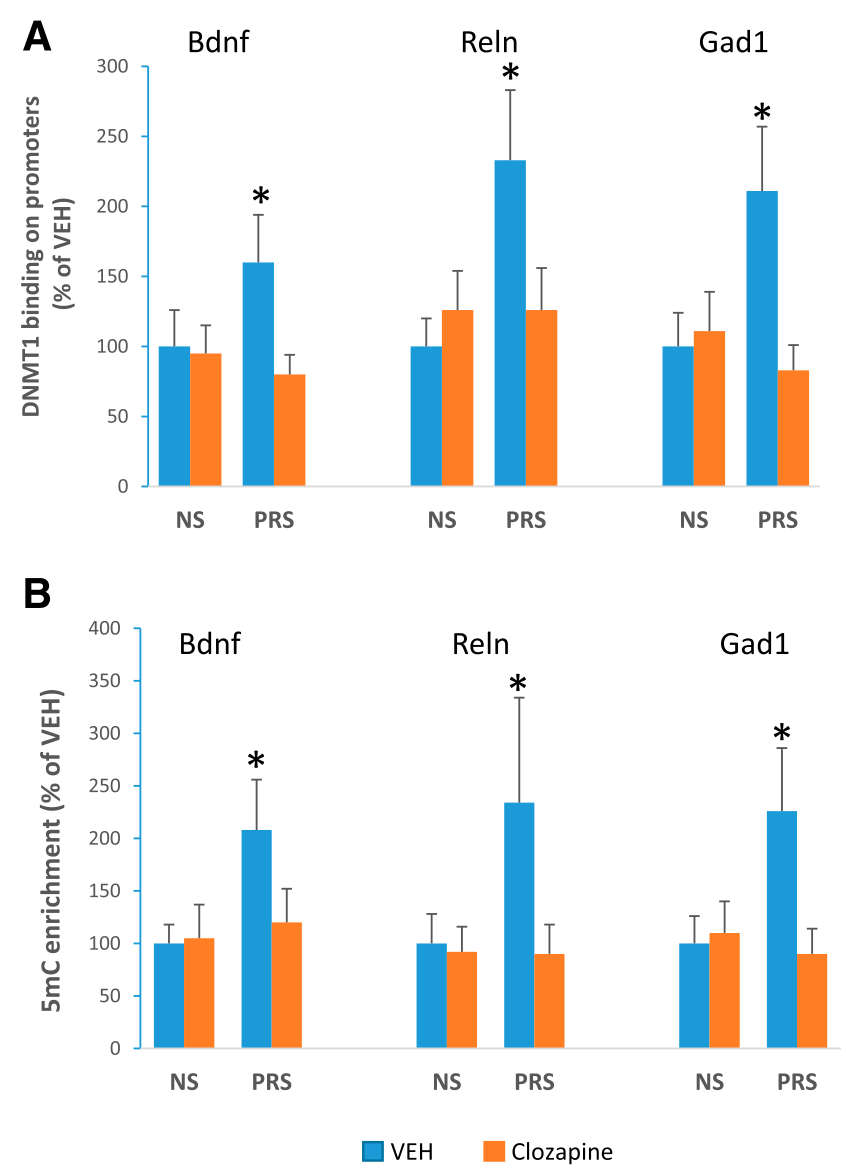

Fig. 3. The increased DNMT1 binding (A) and levels of $5 \mathrm{mC}(\mathrm{B})$ at Bdnf-ix, Reln, and Gad1 promoter regions in the frontal cortex of PRS mice are reversed by treatment with CLO $(5 \mathrm{mg} / \mathrm{kg}$ s.c. twice a day for 5 days $)$. Note that there was no change of $5 \mathrm{mC}$ in the NS mice receiving CLO. The data are expressed as the mean \pm S.D. of five animals per group and analyzed with two-way ANOVA followed by Tukey's multiple-comparisons test. (A) DNMT1 binding to $B d n f-i x\left(F_{1,16}=10.63 P=0.005\right), \operatorname{Reln}\left(F_{1,16}=\right.$ $19.2, P=0.0005)$, and $\operatorname{Gad} 1\left(F_{1,16}=25.26, P=0.0001\right)$. (B) $5 \mathrm{mC}$ enrichment at Bdnf-ix $\left(F_{1,16}=8.36 P=0.01\right)$, Reln $\left(F_{1,16}=7.32, P=0.02\right.$, and Gad1 $\left(F_{1,16}=18.61, P=0.0005\right) . * P<0.05$ when $P R S$ mice are compared with the respective NS VEH-treated mice. $\# P<0.05$ when treated PRS mice are compared with PRS mice receiving VEH.

of CLO with striking behavioral and neuroepigenetic improvements, whereas HAL and RIS fail to do so. The behavioral and molecular effects of CLO are mimicked by the intracerebroventricular injection of the DNMT inhibitor RG108 (Asgatay et al., 2014), suggesting that PRS mice may help to identify novel families of neuroleptic drugs capable of reducing DNA hypermethylation and correcting the behavioral endophenotypes associated with these disorders.

Mice born from mothers stressed during gestation show that, as in humans, environmental insults occurring at critical periods of central nervous system development can trigger epigenetic modifications at psychiatric disorder candidate genes, including Reln, Gad1, and Bdnf, leading to their downregulation (Khashan et al., 2008; van Os et al., 2010; Markham and Koenig 2011; McGowan et al., 2011; Zhang et al., 2013; Cao-Lei et al., 2015, 2016; Scheinost et al., 2017). These epigenetic changes are paralleled by behavioral deficits that are phenotypically reminiscent of those observed in psychiatric diseases (Dong et al., 2016). Hence, the PRS mouse model has value as an experimental epigenetic model for SZ, 

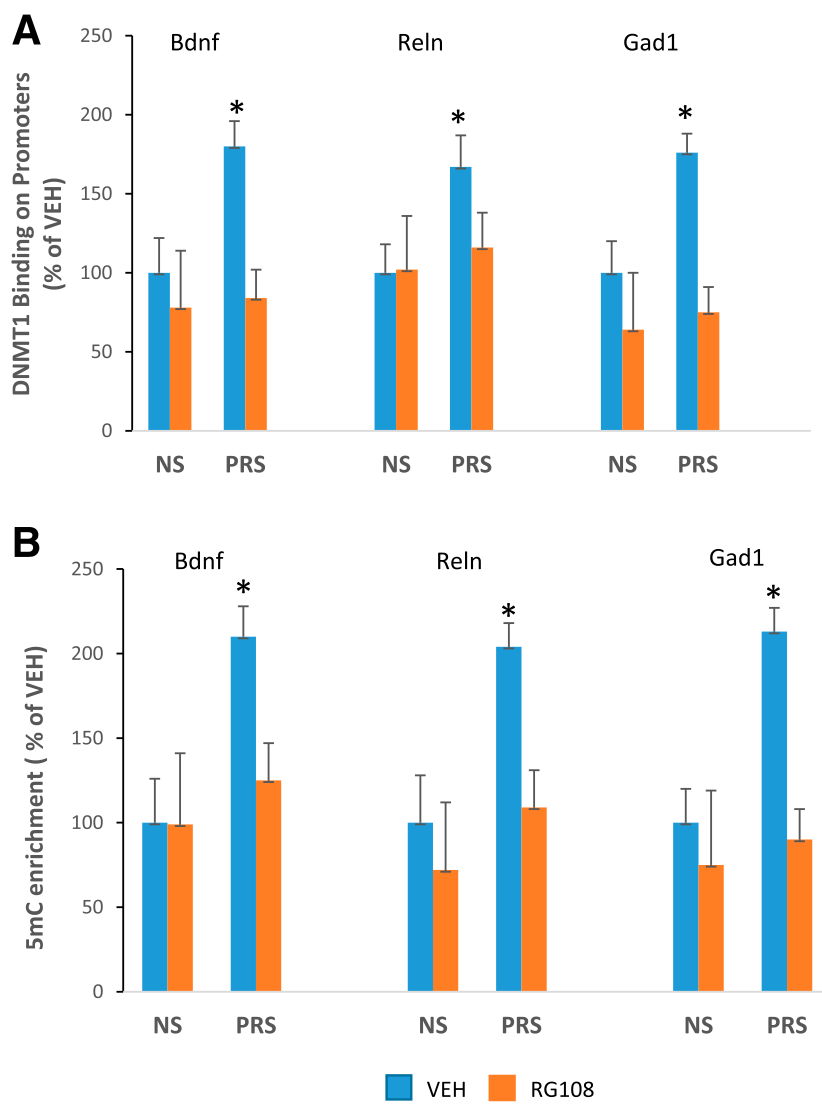

Fig. 4. The increased DNMT1 binding (A) and levels of $5 \mathrm{mC}(\mathrm{B})$ at Bdnf-ix, Reln, and Gad1 promoter regions in the frontal cortex of PRS mice are reversed by treatment with RG108. Note that there is no change of $5 \mathrm{mC}$ in the NS mice receiving RG108. The data are expressed as the mean \pm S.D. of five animals per group and analyzed with two-way ANOVA followed by Tukey's multiple comparisons. (A) DNMT1 binding at Bdnf-ix $\left(F_{1,16}=\right.$ $11.39 P=0.004), \operatorname{Reln}\left(F_{1,16}=6.15, P=0.02\right)$, and $\operatorname{Gad} 1\left(F_{1,16}=9.26, P=\right.$ $0.008) . * P<0.05$ when PRS mice are compared with the respective NS VEH-treated mice. $\# P<0.001$ when treated PRS mice are compared with PRS mice receiving VEH. (B) $5 \mathrm{mc}$ enrichment at Bdnf-ix $\left(F_{1,16}=9.75 P=\right.$ $0.007)$, Reln $\left(F_{1,16}=6.75, P=0.02\right)$, and Gad1 $\left(F_{1,16}=15.94, P=0.001\right)$. $* P<0.05$ when PRS mice are compared with the respective NS VEHtreated mice. $\# P<0.05$ when treated PRS mice are compared with PRS mice receiving $\mathrm{VEH}$.

SZA, and BP disorder and can be useful for screening potential new anti-SZ/SZA/BP disorder drugs for preclinical efficacy and perhaps prophylactic intervention.

Despite the large numbers of preclinical and clinical efforts aimed at delineating the role of major neurotransmitter receptors in the anti-SZ profile of different classes of antipsychotic drugs, little is known regarding the role that these receptors play in the regulation of the neurodevelopmental epigenetic pathology underlying SZ and related psychiatric disorders. Here, we show that by investigating the behavioral and molecular neuroepigenetic alterations induced by typical and atypical neuroleptic drugs (Jarskog et al., 2007), it should be possible to identify or exclude the contribution of each receptor or combination of receptors and their corresponding signal transduction mechanisms to the regulation of altered chromatin remodeling events in PRS mice.

For example, we compared two atypical (CLO and RIS) and one typical (HAL) antipsychotic drug on the behavioral and molecular (chromatin remodeling) alterations observed in PRS mice. We confirmed previous results that doses of HAL

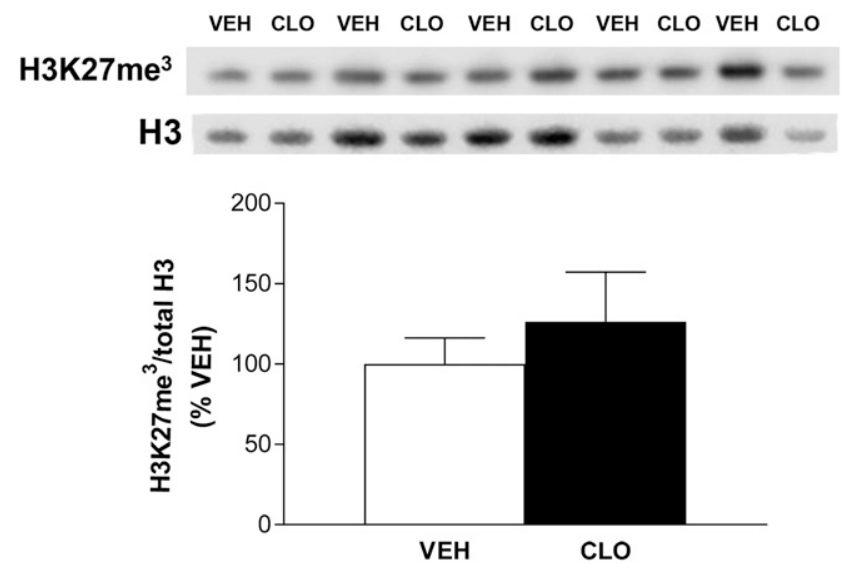

Fig. 5. Effect of CLO treatment on $\mathrm{H} 3 \mathrm{~K} 27 \mathrm{me}^{3}$ protein levels in the frontal cortex of adult male mice. CLO was administered at the dose of $5 \mathrm{mg} / \mathrm{kg}$ twice a day for 5 days. Protein levels of H3K27 $\mathrm{me}^{3}$ are shown as H3K27me3/total H3 ratio. Optical densities are expressed as a percentage of VEH. Data are the mean \pm S.D. of five mice per experimental group. Representative Western blot image is shown on top. Data indicate that there are no significant differences (unpaired $t$ test).

that are capable of blocking DRD2 function and inducing extrapyramidal side effects but do not have serotonin receptor affinity, fail to normalize the altered behavioral endophenotypes or to reduce promoter hypermethylation in PRS mice (Table 1). In contrast, CLO, which binds with high affinity to serotonin receptors but with low affinity to DRD2 (Table 1), normalizes the altered endophenotypes (Fig. 1) and aberrant chromatin remodeling (Figs. 2 and 3). These data suggest that the effects of CLO are not mediated solely by the blockade of DRD2 function.

To further clarify this issue, we studied RIS (an antipsychotic drug with high affinity for both DRD2 and HTR1A, HTR2A, HTR2C, and ADORA1; see Table 1). This atypical antipsychotic drug failed to induce the restorative behavioral and molecular effects reported with CLO (Figs. 1 and 2). The lack of effects of HAL or RIS on the behavioral and molecular neuroepigenetic endophenotypes of PRS mice support the concept that DRD2 as well HTR1A, HTR2A, HTR2C, and ADORA1 are not necessary to explain the corrective action of CLO in our PRS mouse model. However, we cannot rule out a role for CHM1 receptors at this point. The question of whether CLO modulates DNA methylation dynamics bypassing specific neurotransmitter receptors by directly interfering with the binding of DNMT1 to candidate SZ gene promoters or by activating DNA demethylation processes also remains unresolved.

To establish whether the restorative effects of CLO on altered Dnmt1 binding and DNA methylation processes are independent from their action on neurotransmitter receptors and instead dependent on a direct action on DNA methylating and demethylating enzymes and cofactors, we treated mice with RG108, a competitive Dnmt1 antagonist with no known neurotransmitter receptor agonist or antagonist affinity (Asgatay et al., 2014). This drug reduces the increased DNMT1 binding to the Bdnf, Reln, and Gad1 promoters, and also reduces the enrichment of $5 \mathrm{mC}$ to these promoters and restores the behavioral deficits in PRS mice. To explain the behavioral and biochemical action of CLO, we studied whether CLO, like VPA, could alter histone post-transcriptional modifications. In addition, there has been at least one report 
(Huang et al., 2007) showing increased $\mathrm{H} 3 \mathrm{~K} 4 \mathrm{me}^{3}$ in the mouse cerebral cortex after CLO administration ( $5 \mathrm{mg} / \mathrm{kg}$ ) for 21 days. However, no CLO-mediated action on histones was detected with the drugs and doses tested in our study. We also studied the effects of CLO on the expression of DNMTs and TETs (ten 11 translocation family of proteins) and observed that these enzymes are slightly increased in the cortices of PRS mice. CLO normalizes the increased levels in the PRS mice but fails to change the basal level of the enzymes (Dong et al., 2016).

In preliminary studies, we tested whether CLO has Dnmt1 inhibitory activity using purified recombinant protein in vitro. CLO was ineffective at inhibiting Dnmt1 activity up to concentrations of $10^{-3} \mathrm{M}$ (unpublished data). Hence, the mechanisms underlying the marked effects of CLO on DNA methylation are not yet adequately explained.

Overall, the data suggest that drugs or conditions that reduce DNA methylation by reducing DNMT levels, or by blocking DNMT binding or activity to SZ target genes may be desirable or even required, either alone or in association with antipsychotic drugs, to restore normal behavioral activity in PRS mice and likewise to provide beneficial effects to patients with BP disorder, SZA, and SZ who have an underlying epigenetic etiopathogenesis.

The PRS mouse model is insensitive to neuroleptic drugs (i.e., HAL, RIS) that do not have an epigenetic action but is sensitive to drugs like CLO, VPA, (Matrisciano et al., 2013; Dong et al., 2016), and RG108 (present results) because these drugs modulate chromatin function. In this context, PRS mice behave like patients with "antipsychotic-resistant" SZ, predicting treatment responses to CLO and as yet unexplored neuroleptic agents. The above data support our scientific premise that the PRS mouse represents a relevant model for studying interactions between antipsychotic drugs and aberrant epigenetic mechanisms that underlie the behavioral pathology associated with SZ, SZA, and BP disorder.

Collectively, the behavioral and biochemical data with neuroleptic drugs support the hypothesis that the PRS mouse has value to screen for novel antipsychotic drugs with improved clinical efficacy and lower side effect liability. Furthermore, the PRS model theoretically has the potential for predicting treatment responses at specific initial stages of the illness with particular attention to early disease detection and possible prophylactic intervention.

\section{Authorship Contributions}

Participated in research design: Dong, Locci, Grayson, Guidotti.

Conducted experiments: Dong, Locci.

Performed data analysis: Dong, Locci, Gatta.

Wrote or contributed to the writing of the manuscript: Dong, Locci, Gatta, Grayson, Guidotti.

\section{References}

Alachkar A, Wang L, Yoshimura R, Hamzeh AR, Wang Z, Sanathara N, Lee SM, Xu $\mathrm{X}$, Abbott GW, and Civelli O (2018) Prenatal one-carbon metabolism dysregulation programs schizophrenia-like deficits. Mol Psychiatry 23:282-294.

Asgatay S, Champion C, Marloie G, Drujon T, Senamaud-Beaufort C, Ceccaldi A, Erdmann A, Rajavelu A, Schambel P, Jeltsch A, et al. (2014) Synthesis and evaluation of analogues of N-phthaloyl-l-tryptophan (RG108) as inhibitors of DNA methyltransferase 1. J Med Chem 57:421-434.

Bock J, Wainstock T, Braun K, and Segal M (2015) Stress in utero: prenatal programming of brain plasticity and cognition. Biol Psychiatry 78:315-326.

Cao-Lei L, Elgbeili G, Massart R, Laplante DP, Szyf M, and King S (2015) Pregnant women's cognitive appraisal of a natural disaster affects DNA methylation in their children 13 years later: project ice storm. Transl Psychiatry 5:e515.

Cao-Lei L, Veru F, Elgbeili G, Szyf M, Laplante DP, and King S (2016) DNA methylation mediates the effect of exposure to prenatal maternal stress on cytokine production in children at age 131/2 years: Project Ice Storm. Clin Epigenetics 8:54.
Dong E, Dzitoyeva S, Matrisciano F, Tueting P, Grayson DR, and Guidotti A (2015a) Brain-derived neurotrophic factor epigenetic modifications associated with schizophrenia-like phenotype induced by prenatal stress in mice. Biol Psychiatry 77:589-596.

Dong E, Guidotti A, Zhang H, and Pandey SC (2018) Prenatal stress leads to chromatin and synaptic remodeling and excessive alcohol intake comorbid with anxiety-like behaviors in adult offspring. Neuropharmacology 140:76-85.

Dong E, Nelson M, Grayson DR, Costa E, and Guidotti A (2008) Clozapine and sulpiride but not haloperidol or olanzapine activate brain DNA demethylation. Proc Natl Acad Sci USA 105:13614-13619.

Dong E, Ruzicka WB, Grayson DR, and Guidotti A (2015b) DNAmethyltransferase1 (DNMT1) binding to $\mathrm{CpG}$ rich GABAergic and BDNF promoters is increased in the brain of schizophrenia and bipolar disorder patients. Schizophr Res 167:35-41.

Dong E, Tueting P, Matrisciano F, Grayson DR, and Guidotti A (2016) Behavioral and molecular neuroepigenetic alterations in prenatally stressed mice: relevance for the study of chromatin remodeling properties of antipsychotic drugs. Transl Psychiatry 6:e711.

Fatemi SH and Folsom TD (2009) The neurodevelopmental hypothesis of schizophrenia, revisited. Schizophr Bull 35:528-548.

Fine R, Zhang J, and Stevens HE (2014) Prenatal stress and inhibitory neuron systems: implications for neuropsychiatric disorders. Mol Psychiatry 19:641-651.

Gatta E, Auta J, Gavin DP, Bhaumik DK, Grayson DR, Pandey SC, and Guidotti A (2017) Emerging role of one-carbon metabolism and DNA methylation enrichment on $\delta$-containing GABAA receptor expression in the cerebellum of subjects with alcohol use disorders (AUD). Int J Neuropsychopharmacol 20:1013-1026.

Grayson DR and Guidotti A (2013) The dynamics of DNA methylation in schizophrenia and related psychiatric disorders. Neuropsychopharmacology 38:138-166

Grayson DR and Guidotti A (2016) Merging data from genetic and epigenetic approaches to better understand autistic spectrum disorder. Epigenomics 8:85-104.

Guidotti A, Auta J, Chen Y, Davis JM, Dong E, Gavin DP, Grayson DR, Matrisciano F, Pinna G, Satta R, et al. (2011) Epigenetic GABAergic targets in schizophrenia and bipolar disorder. Neuropharmacology 60:1007-1016.

Guidotti A, Dong E, Kundakovic M, Satta R, Grayson DR, and Costa E (2009) Characterization of the action of antipsychotic subtypes on valproate-induced chromatin remodeling. Trends Pharmacol Sci 30:55-60.

Huang HS, Matevossian A, Whittle C, Kim SY, Schumacher A, Baker SP, and Akbarian S (2007) Prefrontal dysfunction in schizophrenia involves mixedlineage leukemia 1-regulated histone methylation at GABAergic gene promoters. J Neurosci 27:11254-11262.

Impagnatiello F, Pesold C, Longone $\mathrm{P}$, Caruncho H, Fritschy JM, Costa E, and Guidotti A (1996) Modifications of gamma-aminobutyric acidA receptor subunit expression in rat neocortex during tolerance to diazepam. Mol Pharmacol 49 822-831.

Jarskog LF, Miyamoto S, and Lieberman JA (2007) Schizophrenia: new pathological insights and therapies. Annu Rev Med 58:49-61.

Jones CA, Watson DJG, and Fone KCF (2011) Animal models of schizophrenia. Br J Pharmacol 164:1162-1194.

Khashan AS, Abel KM, McNamee R, Pedersen MG, Webb RT, Baker PN, Kenny LC, and Mortensen PB (2008) Higher risk of offspring schizophrenia following antenatal maternal exposure to severe adverse life events. Arch Gen Psychiatry 65: $146-152$

Labouesse MA, Dong E, Grayson DR, Guidotti A, and Meyer U (2015) Maternal immune activation induces GAD1 and GAD2 promoter remodeling in the offspring prefrontal cortex. Epigenetics 10:1143-1155.

Lisman JE, Coyle JT, Green RW, Javitt DC, Benes FM, Heckers S, and Grace AA (2008) Circuit-based framework for understanding neurotransmitter and risk gene interactions in schizophrenia. Trends Neurosci 31:234-242.

Markham JA and Koenig JI (2011) Prenatal stress: role in psychotic and depressive diseases. Psychopharmacology (Berl) 214:89-106.

Matrisciano F, Tueting P, Dalal I, Kadriu B, Grayson DR, Davis JM, Nicoletti F, and Guidotti A (2013) Epigenetic modifications of GABAergic interneurons are associated with the schizophrenia-like phenotype induced by prenatal stress in mice. Neuropharmacology 68:184-194.

McGowan PO, Suderman M, Sasaki A, Huang TC, Hallett M, Meaney MJ, and Szyf M (2011) Broad epigenetic signature of maternal care in the brain of adult rats. PLoS One 6:e14739.

Meltzer HY (2012) Serotonergic mechanisms as targets for existing and novel antipsychotics. Handb Exp Pharmacol 212:87-124.

Mill J, Tang T, Kaminsky Z, Khare T, Yazdanpanah S, Bouchard L, Jia P, Assadzadeh A, Flanagan J, Schumacher A, et al. (2008) Epigenomic profiling reveals DNAmethylation changes associated with major psychosis. Am J Hum Genet 82:696-711.

Murmu MS, Salomon S, Biala Y, Weinstock M, Braun K, and Bock J (2006) Changes of spine density and dendritic complexity in the prefrontal cortex in offspring of mothers exposed to stress during pregnancy. Eur $J$ Neurosci 24:1477-1487.

Ptak C and Petronis A (2010) Epigenetic approaches to psychiatric disorders. Dialogues Clin Neurosci 12:25-35.

Ruzicka WB, Subburaju S, and Benes FM (2015) Circuit-and diagnosis-specific DNA methylation changes at $\gamma$-aminobutyric acid-related genes in postmortem human hippocampus in schizophenia and bipolar disorder. JAMA Psychiatry 72: 541-551.

Scheinost D, Sinha R, Cross SN, Kwon SH, Sze G, Constable RT, and Ment LR (2017) Does prenatal stress alter the developing connectome? Pediatr Res 81:214-226.

Schizophenia Working Group of the Psychiatric Genomics Consortium (2014). Biological insights from 108 schizophenia-associated genetic loci. Nature 511:421-427.

Sekar A, Bialas AR, de Rivera H, Davis A, Hammond TR, Kamitaki N, Tooley K, Presumey J, Baum M, Van Doren V, et al.; Schizophrenia Working Group of the Psychiatric Genomics Consortium (2016) Schizophrenia risk from complex variation of complement component 4. Nature 530:177-183. 
Simonini MV, Camargo LM, Dong E, Maloku E, Veldic M, Costa E, and Guidotti A (2006) The benzamide MS-275 is a potent, long-lasting brain regionselective inhibitor of histone deacetylases. Proc Natl Acad Sci USA 103 : 1587-1592.

Tremolizzo L, Doueiri MS, Dong E, Grayson DR, Davis J, Pinna G, Tueting P, Rodriguez-Menendez V, Costa E, and Guidotti A (2005) Valproate corrects the schizophrenia-like epigenetic behavioral modifications induced by methionine in mice. Biol Psychiatry 57:500-509.

van Os J, Kenis G, and Rutten BPF (2010) The environment and schizophrenia Nature 468:203-212.

Weinberger DR (1987) Implications of normal brain development for the pathogenesis of schizophrenia. Arch Gen Psychiatry 44:660-669.
Wen L, Li X, Yan L, Tan Y, Li R, Zhao Y, Wang Y, Xie J, Zhang Y, Song C, et al. (2014) Whole-genome analysis of 5-hydroxymethylcytosine and 5-methylcytosine at base resolution in the human brain. Genome Biol 15:R49.

Zhang TY, Labonté B, Wen XL, Turecki G, and Meaney MJ (2013) Epigenetic mechanisms for the early environmental regulation of hippocampal glucocorticoid receptor gene expression in rodents and humans. Neuropsychopharmacology 38: 111-123.

Address correspondence to: Alessandro Guidotti, University of Illinois College of Medicine, Psychiatric Institute, 1601 West Taylor Street, Chicago, IL 60612. E-mail: AGuidotti@psych.uic.edu 